\title{
Enzyme Kinetic Equations of Irreversible and Reversible Reactions in Metabolism
}

\author{
Santiago Imperial, Josep J. Centelles \\ Departament de Bioquímica i Biologia Molecular (Biologia), Facultat de Biologia, Universitat de Barcelona, \\ Barcelona, Spain \\ Email: josepcentelles@ub.edu
}

Received March 2014

\begin{abstract}
This paper compares the irreversible and reversible rate equations from several uni-uni kinetic mechanisms (Michaelis-Menten, Hill and Adair equations) and bi-bi mechanisms (single- and doubledisplacement equations). In reversible reactions, Haldane relationship is considered to be identical for all mechanisms considered and reversible equations can be also obtained from this relationship. Some reversible reactions of the metabolism are also presented, with their equilibrium constant.
\end{abstract}

\section{Keywords}

\author{
Adair Equation, Enzyme Kinetics, Equilibrium Constant, Haldane Relationship, Hill Equation, \\ Metabolism, Michaelis Menten Equation, Reversible Reactions
}

\section{Introduction}

Thermodinamical considerations in a metabolic pathway include different aspects like kinetic analysis, and identification of reversible steps in this pathway [1]. Although most of the reactions are reversible, it is usual in general Biochemistry textbooks to present to students kinetic irreversible equations. For instance, the irreversible Michaelis-Menten equation is a well-known example and it is presented in this way in general Biochemistry books (either to simplify the mechanism, or because this reaction is used for an in vitro study in absence of the product of the reaction). Nevertheless, when performing an in vivo study, or when using a biochemical mathematical model presenting several reactions of a metabolic pathway, reversible equations should be considered. In this paper, we present several reversible equations and we compare them with the irreversible ones.

Haldane relationship, an equation which can only be used for reversible reactions, connects biochemical thermodynamics and biochemical kinetics. Thus, for a reversible uni-uni reaction $\mathrm{A}=\mathrm{P}$, Haldane relationship connects equilibrium constant $K_{e q}$ with kinetic parameters for both irreversible reactions, $A \rightarrow P\left(V_{f}\right.$ and $\left.K_{m A}\right)$ and $\mathrm{P} \rightarrow \mathrm{A}\left(\mathrm{V}_{\mathrm{r}}\right.$ and $\left.\mathrm{K}_{\mathrm{mP}}\right)$. Haldane relationship is in this case: $\mathrm{K}_{\mathrm{eq}}=\mathrm{V}_{\mathrm{f}} \mathrm{K}_{\mathrm{mP}} / \mathrm{V}_{\mathrm{r}} \mathrm{K}_{\mathrm{mA}}$. This is a general relationship that is also valid for several other mechanisms, including Hill equation (although $[\mathrm{P}]_{0.5}$ and $[\mathrm{S}]_{0.5}$ should replace the values of $\mathrm{K}_{\mathrm{mP}}$ and $\mathrm{K}_{\mathrm{mA}}$, respectively). Several reversible equations are obtained from the Haldane relationship considering that in equilibrium total velocity should be zero, and that $\mathrm{V}=\mathrm{V}_{\mathrm{A}_{\rightarrow} \mathrm{P}}-\mathrm{V}_{\mathrm{P}_{\rightarrow} \mathrm{A}}$. Nevertheless, this relationship is not considered universal, as when considering a bi-bi reaction with two reactions: $\mathrm{A}=\mathrm{Q}$, fol- 
lowed by $\mathrm{B}=\mathrm{P}$, Haldane relationship will be the product of the two equilibrium constants:

$\mathrm{K}_{\mathrm{eq}}=\mathrm{K}_{\mathrm{eq}(1)} \mathrm{K}_{\mathrm{eq}(2)}=\left(\mathrm{V}_{\mathrm{f}} \mathrm{K}_{\mathrm{mQ}} / \mathrm{V}_{\mathrm{r}} \mathrm{K}_{\mathrm{mA}}\right)\left(\mathrm{V}_{\mathrm{f}} \mathrm{K}_{\mathrm{mP}} / \mathrm{V}_{\mathrm{r}} \mathrm{K}_{\mathrm{mB}}\right)=\mathrm{V}_{\mathrm{f}}^{2} \mathrm{~K}_{\mathrm{mP}} \mathrm{K}_{\mathrm{mQ}} / \mathrm{V}_{\mathrm{r}}^{2} \mathrm{~K}_{\mathrm{mA}} \mathrm{K}_{\mathrm{mB}}$.

In general, Haldane relationship for a bi-bi mechanism is an equation more similar to the uni-uni equation:

$\mathrm{K}_{\mathrm{eq}}=\mathrm{V}_{\mathrm{f}} \mathrm{K}_{\mathrm{mP}} \mathrm{K}_{\mathrm{mQ}} / \mathrm{V}_{\mathrm{r}} \mathrm{K}_{\mathrm{mA}} \mathrm{K}_{\mathrm{mB}}$.

\section{Kinetic Equations of Reversible Reactions}

\subsection{Uni-Uni Mechanisms}

The easiest mechanism for uni-uni enzyme kinetics is the Michaelis-Menten mechanism. The best known equation is the irreversible equation, which is used for a reaction with one substrate, independently on if the obtained products are one, two, or several. For a uni-uni mechanism, it can be observed a competitive inhibition by the product, as both the substrate and the product bind to the active site. This is the reason for the presence of a term depending on $[\mathrm{P}]$ at the denominator of the reversible equation, which is not considered for an irreversible equation, as $[\mathrm{P}]=0$.

Figure 1 shows the different kinetic equations obtained for several uni-uni mechanisms. Reversible Michaelis-Menten equation is considered as a uni-uni reaction, where $\alpha$ and $\pi$ are the relative concentrations for the substrate and the product respectively: $\alpha=[\mathrm{A}] / \mathrm{K}_{\mathrm{mA}}$ and $\pi=[\mathrm{P}] / \mathrm{K}_{\mathrm{mP}}$.

Although irreversible Hill equation is presented as a general equation (where $h$ is the Hill coefficient), the Adair irreversible equation is presented only for an enzyme with two active centers. Figure 1 also shows the equation obtained for two enzymes (with different kinetic parameters) acting in the same reaction. This could be the case of two isoenzymes. This equation for two enzymes is the same as Adair equation for two sites, as two enzymes have also two active sites. Reversible Hill equation is very similar to Adair equation [2], as it can be calculated from Figure 1 for $h=2$.

It should be noted that the reversible equations should be always converted into irreversible equations considering zero the products concentrations. Thus, Michaelis-Menten and Hill irreversible equations are obtained from the reversible equations on Figure 1 considering $\pi=0$, and Adair irreversible equation is obtained from

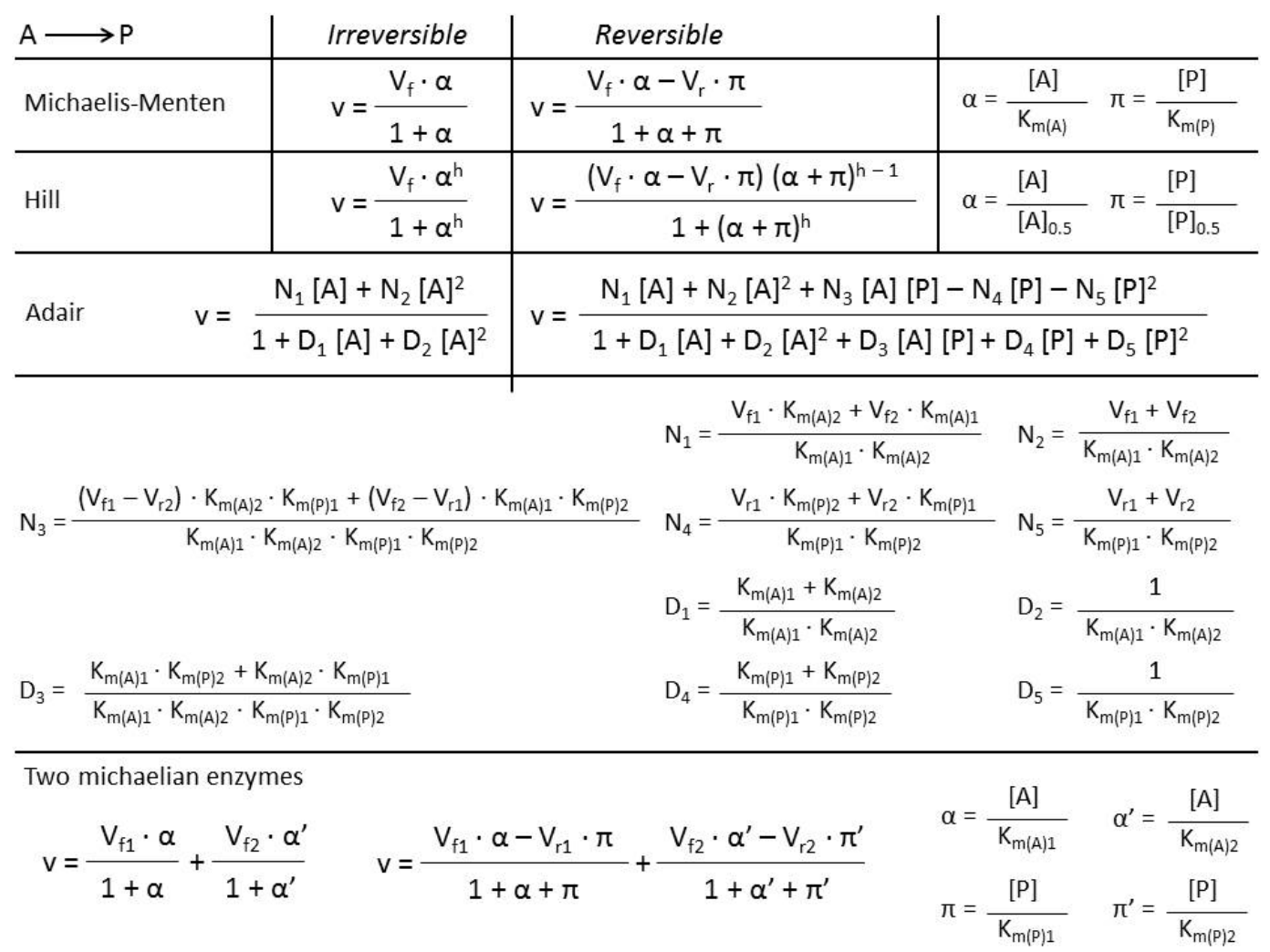

Figure 1. Comparison between the reversible and the irreversible kinetic equations of an uni-uni reaction. 
the reversible equation by considering $[\mathrm{P}]=0$.

Similar equations should be also obtained for the inverse irreversible reaction $(\mathrm{P} \rightarrow \mathrm{A})$ by considering $[\mathrm{A}]=0$ (or $\alpha=0$ ) although in these cases a negative equation is obtained, as the velocity is considered in the inverse sense.

Haldane relationship from these reversible equations can be solved by considering that equilibrium concentrations would led to $\mathrm{v}=0$ (for Michaelis-Menten and Hill equations, $\mathrm{V}_{\mathrm{f}} \alpha_{\mathrm{eq}}=\mathrm{V}_{\mathrm{r}} \pi_{\mathrm{eq}}$ ), and $\mathrm{K}_{\mathrm{eq}}=[\mathrm{P}]_{\mathrm{eq}} /[\mathrm{A}]_{\mathrm{eq}}$. Thus, resulting Haldane relationship to be: $\mathrm{K}_{\mathrm{eq}}=\mathrm{V}_{\mathrm{f}} \mathrm{K}_{\mathrm{mP}} / \mathrm{V}_{\mathrm{r}} \mathrm{K}_{\mathrm{mA}}$.

\subsection{Bi-Bi Mechanisms}

Although there are several possible mechanisms, the most common bi-bi mechanisms include the ternary complex mechanism (random or ordered bi-bi) and the substituted-enzyme mechanism (ping-pong bi-bi). As it can be seen in Figure 2, the main difference between both mechanisms is the independent term present in the denominator of the ternary complex mechanism. This independent term is present either for the irreversible or the reversible equation.

These equations are more complex than those for the uni-uni mechanism, but it should be observed that both irreversible equations can be simplified to a Michaelis-Menten uni-uni equation by considering saturated the concentration of one of the substrates. For example, for a high $\beta, \mathrm{v}=\mathrm{V}_{\mathrm{f}} \alpha \beta /(\beta+\alpha \beta)$, and simplifying, $\mathrm{v}=\mathrm{V}_{\mathrm{f}} \alpha /(1$ $+\alpha)$. Similarly, for a high $\alpha$, the equation obtained would be $\mathrm{v}=\mathrm{V}_{\mathrm{f}} \beta /(1+\beta)$.

The reversible equation, when considering high $\beta$, is transformed to an irreversible Michaelis-Menten equation, with inhibitions of products (P and Q). These inhibitions depend on the bi-bi mechanism considered, and they follow the Cleland laws.

Haldane relationship from these equations can be solved by considering that equilibrium concentrations would led to $\mathrm{v}=0$ (in both cases, $\mathrm{V}_{\mathrm{f}} \alpha_{\mathrm{eq}} \beta_{\mathrm{eq}}=\mathrm{V}_{\mathrm{r}} \pi_{\mathrm{eq}} \rho_{\mathrm{eq}}$ ), and $\mathrm{K}_{\mathrm{eq}}=[\mathrm{P}]_{\mathrm{eq}}[\mathrm{Q}]_{\mathrm{eq}} /[\mathrm{A}]_{\text {eq }}[\mathrm{B}]_{\text {eq }}$. Thus, resulting Haldane relationship would be: $\mathrm{K}_{\mathrm{eq}}=\mathrm{V}_{\mathrm{f}} \mathrm{K}_{\mathrm{mP}} \mathrm{K}_{\mathrm{mQ}} / \mathrm{V}_{\mathrm{r}} \mathrm{K}_{\mathrm{mA}} \mathrm{K}_{\mathrm{mB}}$.

\subsection{Multisubstrate Mechanisms}

We have considered until now uni-uni and bi-bi mechanism. Nevertheless, some reactions can be also uni-bi, or bi-uni. Irreversible equations from uni-uni and from uni-bi are identical, as both consider only one substrate. But reversible reactions present other factors in the numerators, as it can be seen in Figure $\mathbf{1}$ and Figure 2. Thus, for uni-bi reactions, numerators of the equations can be easily corrected by using the uni positive factor and the bi negative factor in the numerator: $\mathrm{V}_{\mathrm{f}} \alpha-\mathrm{V}_{\mathrm{r}} \pi \rho$. And in the same way, the bi-uni equation should have the following factor as numerator: $\mathrm{V}_{\mathrm{f}} \alpha \beta-\mathrm{V}_{\mathrm{r}} \pi$. In general, numerators can be deduced from the previous Figures. In fact, if the reaction mechanism is known, the King and Altman method [3] can be used to deduce the enzymatic equation. This method can be easily performed from the web page http://biokin.com/king-altman/index.html .

\section{Some Examples of Reversible Reactions in Metabolism}

Equilibrium constant can be used to calculate kinetic parameters by using Haldane relationship. For this reason, we present in Table 1 a brief summary of some reversible reactions extracted from Barman [4]. Equilibrium constants from the table are not taken all in the same conditions of $\mathrm{pH}$ and temperature, and the substrate or products concentrations in the cell would indicate whether the reaction is far or near equilibrium. Reversible

\begin{tabular}{l|c|c}
$\mathrm{A}+\mathrm{B} \longrightarrow \mathrm{P}+\mathrm{Q}$ & Irreversible & Reversible \\
\hline $\begin{array}{c}\text { Ternary complex } \\
\text { mechanism }\end{array}$ & $\mathrm{v}=\frac{\mathrm{V}_{\mathrm{f}} \cdot \alpha \beta}{1+\alpha+\beta+\alpha \beta}$ & $v=\frac{\mathrm{V}_{\mathrm{f}} \cdot \alpha \beta-\mathrm{V}_{\mathrm{r}} \cdot \pi \rho}{1+\alpha+\beta+\pi+\rho+\alpha \beta+\alpha \pi+\beta \rho+\pi \rho+\alpha \beta \pi+\beta \pi \rho}$ \\
\hline $\begin{array}{c}\text { Substituted-enzyme } \\
\text { mechanism }\end{array}$ & $\mathrm{v}=\frac{\mathrm{V}_{\mathrm{f}} \cdot \alpha \beta}{\alpha+\beta+\alpha \beta}$ & $\mathrm{v}=\frac{\mathrm{V}_{\mathrm{f}} \cdot \alpha \beta-\mathrm{V}_{\mathrm{r}} \cdot \pi \rho}{\alpha+\beta+\pi+\rho+\alpha \beta+\alpha \pi+\beta \rho+\pi \rho}$ \\
\hline & $\alpha=\frac{[\mathrm{A}]}{\mathrm{K}_{\mathrm{m}(\mathrm{A})}} \quad \beta=\frac{[\mathrm{B}]}{\mathrm{K}_{\mathrm{m}(\mathrm{B})}} \quad \pi=\frac{[\mathrm{P}]}{\mathrm{K}_{\mathrm{m}(\mathrm{P})}} \quad \rho=\frac{[Q]}{\mathrm{K}_{m(\mathrm{O})}}$
\end{tabular}

Figure 2. Comparison between the reversible and irreversible kinetic equations of a bi-bi reaction. 
Table 1. Some reversible reactions of the most common pathways in metabolism (extracted from [4]).

\begin{tabular}{|c|c|c|}
\hline \multirow{2}{*}{$\begin{array}{c}\text { Enzyme } \\
\text { (E.C. number) }\end{array}$} & \multirow[b]{2}{*}{ Reaction } & \multirow[b]{2}{*}{$\mathbf{K}_{\mathrm{eq}}$} \\
\hline & & \\
\hline $\begin{array}{l}\text { Alcohol dehydrogenase } \\
\text { (EC 1.1.1.1.) }\end{array}$ & alcohol $+\mathrm{NAD}=$ aldehyde or ketone + reduced NAD & $8.0 \times 10^{-12}$ \\
\hline $\begin{array}{c}\text { Glycerol-3-phosphate } \\
\text { dehydrogenase (EC 1.1.1.8.) }\end{array}$ & L-glycerol-3-P + NAD = dihydroxyacetone phosphate + reduced NAD & $1.010^{-12}$ \\
\hline $\begin{array}{l}\text { Lactate dehydrogenase } \\
\text { (EC 1.1.1.27.) }\end{array}$ & L-lactate $+\mathrm{NAD}=$ pyruvate + reduced NAD & $2.76 \times 10^{-6}$ \\
\hline $\begin{array}{l}\text { Malate dehydrogenase } \\
\text { (EC 1.1.1.37.) }\end{array}$ & L-malate $+\mathrm{NAD}=$ oxaloacetate + reduced NAD & $6.4 \times 10^{-13}$ \\
\hline $\begin{array}{c}\text { Glucose 6-phosphate } \\
\text { dehydrogenase (EC 1.1.1.49.) }\end{array}$ & D-glucose 6-phosphate + NADP = D-glucono- $\delta$-lactone 6-phosphate + reduced NADP & $6.0 \times 10^{-7}$ \\
\hline $\begin{array}{c}\text { Glyceraldehyde-phosphate } \\
\text { dehydrogenase (EC 1.2.1.12.) }\end{array}$ & D-glyceraldehyde-3-phosphate + Pi + NAD = 1,3-diphospho-D-glycerate + reduced NAD & 0.5 \\
\hline $\begin{array}{l}\text { Butyryl-CoA dehydrogenase } \\
\text { (EC 1.3.99.2.) }\end{array}$ & butyryl-CoA + FAD = crotonoyl-CoA + FADH ${ }_{2}$ & 0.22 \\
\hline $\begin{array}{l}\text { Alanine dehydrogenase } \\
\text { (EC 1.4.1.1.) }\end{array}$ & L-alanine $+\mathrm{H}_{2} \mathrm{O}+\mathrm{NAD}=$ pyruvate $+\mathrm{NH}_{3}+$ reduced $\mathrm{NAD}$ & $6.98 \times 10^{-14}$ \\
\hline $\begin{array}{l}\text { Glutamate dehydrogenase } \\
\text { (EC 1.4.1.2.) }\end{array}$ & L-glutamate $+\mathrm{H}_{2} \mathrm{O}+\mathrm{NAD}=2$-oxoglutarate $+\mathrm{NH}_{3}+$ reduced $\mathrm{NAD}$ & $4.5 \times 10^{-14}$ \\
\hline $\begin{array}{l}\text { Tetrahydrofolate dehydrogenase } \\
\text { (EC 1.5.1.3.) }\end{array}$ & 5,6,7,8-tetrahydrofolate + NADP = 7,8-dihydrofolate + reduced NADP & $1.7910^{-12}$ \\
\hline $\begin{array}{l}\text { NAD(P) transhydrogenase } \\
\text { (EC 1.6.1.1.) }\end{array}$ & reduced NADP + NAD = NADP + reduced NAD & 1.43 \\
\hline $\begin{array}{c}\text { Glutathione reductase } \quad \text { (EC } \\
\text { 1.6.4.2.) }\end{array}$ & reduced $\mathrm{NAD}(\mathrm{P})+$ oxidized glutathione $=\mathrm{NAD}(\mathrm{P})+2$ glutathione & $9.8 \times 10^{-6}$ \\
\hline $\begin{array}{c}\text { Serine } \\
\text { hydroxymethyltransferase } \\
\text { (EC } 2.1 .2 .1 .)\end{array}$ & L-serine + tetrahydrofolate $=$ glycine $+5,10$-methylenetetrahydrofolate & 10.2 \\
\hline $\begin{array}{l}\text { Methylmalonyl-CoA } \\
\text { carboxyltransferase } \\
\text { (EC 2.1.3.1.) }\end{array}$ & methylmalonyl-CoA + pyruvate $=$ propionyl-CoA + oxaloacetate & 0.526 \\
\hline $\begin{array}{l}\text { Ornithine carbamoyltransferase } \\
\text { (EC 2.1.3.3.) }\end{array}$ & carbamoylphosphate + L-ornithine $=\mathrm{Pi}+$ L-citrulline & $1 \times 10^{5}$ \\
\hline Transketolase (EC 2.2.1.1.) & sedoheptulose 7-P + D-glyceraldehyde 3-P = D-ribose 5-P + D-xylulose 5-P & 0.95 \\
\hline Transaldolase (EC 2.2.1.2.) & sedoheptulose 7-P + D-glyceraldehyde 3-P = D-erythrose 4-P + D-fructose 6-P & 1.05 \\
\hline $\begin{array}{l}\text { Choline acetyltransferase } \\
\text { (EC 2.3.1.6) }\end{array}$ & acetyl-CoA + choline $=$ CoA + acetylcholine & $5.1 \times 10^{3}$ \\
\hline $\begin{array}{c}\text { Carnitine acyltransferase } \\
\text { (EC 2.3.1.7.) }\end{array}$ & acetyl-CoA + carnitine $=$ CoA + acetylcarnitine & 1.67 \\
\hline $\begin{array}{c}\text { Phosphate acyltransferase } \\
\text { (EC 2.3.1.8.) }\end{array}$ & acetyl-CoA $+\mathrm{P}_{\mathrm{i}}=\mathrm{CoA}+$ acetylphosphate & $1.35 \times 10^{-2}$ \\
\hline $\begin{array}{c}\text { Acetyl-CoA acetyltransferase } \\
\text { (EC 2.3.1.9.) }\end{array}$ & 2 acetyl-CoA = CoA + acetoacetyl-CoA & $\sim 2 \times 10^{-5}$ \\
\hline $\begin{array}{c}\text { Adenine } \\
\text { phosphoribosyltransferase } \\
\text { (EC 2.4.2.7.) }\end{array}$ & AMP + pyrophosphate $=$ adenine +5 -phospho- $\alpha$-ribosyl-pyrophosphate & 0.1 \\
\hline $\begin{array}{l}\text { Aspartate aminotransferase } \\
\text { (EC 2.6.1.1.) }\end{array}$ & L-aspartate +2 -oxoglutarate $=$ oxaloacetate + L-glutamate & $0.16-0.17$ \\
\hline $\begin{array}{c}\text { Alanine aminotransferase } \\
\text { (EC 2.6.1.2.) }\end{array}$ & L-alanine +2 -oxoglutarate $=$ pyruvate + L-glutamate & 2.2 \\
\hline Glucokinase (EC 2.7.1.2.) & ATP + D-glucose = ADP + D-glucose-6-phosphate & $3.86 \times 10^{2}$ \\
\hline
\end{tabular}




\section{Continued}

\begin{tabular}{|c|c|c|}
\hline Galactokinase (EC 2.7.1.6.) & ATP + D-galactose $=$ ADP + D-galactose-1-phosphate & 26 \\
\hline Pyruvate kinase (EC 2.7.1.40.) & ATP + pyruvate $=$ ADP + phosphoenolpyruvate & $1.55 \times 10^{-4}$ \\
\hline Acetate kinase (EC 2.7.2.1.) & $\mathrm{ATP}+$ acetate $=\mathrm{ADP}+$ acetylphosphate & $\sim 8 \times 10^{-3}$ \\
\hline Carbamate kinase (EC 2.7.2.2.) & ATP $+\mathrm{NH}_{3}+\mathrm{CO}_{2}=\mathrm{ADP}+$ carbamoylphosphate & $410^{-2}$ \\
\hline $\begin{array}{c}\text { Phosphoglycerate kinase } \\
\text { (EC 2.7.2.3.) }\end{array}$ & ATP + 3-phospho-D-glycerate = ADP + 1,3-diphospho-D-glycerate & $2.9 \times 10^{-4}$ \\
\hline Creatine kinase (EC 2.7.3.2.) & $\mathrm{ATP}+$ creatine $=\mathrm{ADP}+$ phosphocreatine & $7.2 \times 10^{-9}$ \\
\hline Adenylate kinase (EC 2.7.4.3.) & $\mathrm{ATP}+\mathrm{AMP}=2 \mathrm{ADP}$ & 2.26 \\
\hline Glutaminase (EC 3.5.1.2.) & L-glutamine $+\mathrm{H}_{2} \mathrm{O}=$ L-glutamate $+\mathrm{NH}_{3}$ & 320 \\
\hline $\begin{array}{l}\text { Dihydropyrimidinase } \\
\text { (EC 3.5.2.2.) }\end{array}$ & 4,5-dihydrouracil $+\mathrm{H}_{2} \mathrm{O}=$ 3-ureidopropionate & 0.67 \\
\hline Dihydro-orotase (EC 3.5.2.3.) & L-4,5-dihydro-orotate $+\mathrm{H}_{2} \mathrm{O}=\mathrm{N}$-carbamoyl-L-aspartate & 1.9 \\
\hline $\begin{array}{l}\text { Methenyltetrahydrofolate } \\
\text { cyclohydrolase (EC 3.5.4.9.) }\end{array}$ & 5,10-methyltetrahydrofolate $+\mathrm{H}_{2} \mathrm{O}=10$-formyltetrahydrofolate & $2.4 \times 10^{-8}$ \\
\hline $\begin{array}{c}\text { Phosphopyruvate carboxylase } \\
\text { (EC 4.1.1.32.) }\end{array}$ & $\mathrm{GTP}+$ oxaloacetate $=\mathrm{GDP}+$ phosphoenolpyruvate $+\mathrm{CO}_{2}$ & 0.372 \\
\hline $\begin{array}{l}\text { Fructosediphosphate aldolase } \\
\text { (EC 4.1.2.13.) }\end{array}$ & fructose-1,6-diphosphate = dihydroxyacetone-P + D-glyceraldehyde-3-P & $8.1 \times 10^{-5}$ \\
\hline Citrate lyase (EC 4.1.3.6.) & citrate $=$ acetate + oxaloacetate & 0.325 \\
\hline Citrate synthase (EC 4.1.3.7.) & citrate $+\mathrm{CoA}=$ acetyl-CoA $+\mathrm{H}_{2} \mathrm{O}+$ oxaloacetate & $1.2 \times 10^{-4}$ \\
\hline ATP citrate lyase (EC 4.1.3.8.) & $\mathrm{ATP}+$ citrate $+\mathrm{CoA}=\mathrm{ADP}+\mathrm{P}_{\mathrm{i}}+$ acetyl-CoA + oxaloacetate & $1.0-1.5$ \\
\hline Carbonic anhydrase (EC 4.2.1.1.) & $\mathrm{H}_{2} \mathrm{CO}_{3}=\mathrm{CO}_{2}+\mathrm{H}_{2} \mathrm{O}$ & $2.51 \times 10^{6}$ \\
\hline Fumarate hydratase (EC 4.2.1.2.) & L-malate $=$ fumarate $+\mathrm{H}_{2} \mathrm{O}$ & 0.23 \\
\hline $\begin{array}{l}\text { Enoyl-CoA hydratase } \\
\text { (EC 4.2.1.1.7) }\end{array}$ & L-3-hydroxyacil-Coa $=$ crotonoyl-CoA $+\mathrm{H}_{2} \mathrm{O}$ & 16.2 \\
\hline $\begin{array}{c}\text { Argininosuccinate lyase } \\
\text { (EC 4.3.2.1.) }\end{array}$ & L-argininosuccinate $=$ fumarate + L-arginine & $1.14 \times 10^{-2}$ \\
\hline $\begin{array}{l}\text { Adenylosuccinate lyase } \\
\text { (EC 4.3.2.2.) }\end{array}$ & adenylosuccinate $=$ fumarate + AMP & $6.8 \times 10^{-3}$ \\
\hline $\begin{array}{l}\text { Glutamate racemase } \\
\text { (EC 5.1.1.3.) }\end{array}$ & L-glutamate = D-glutamate & $\sim 1$ \\
\hline $\begin{array}{l}\text { Hydroxyproline epimerase } \\
\text { (EC 5.1.1.8.) }\end{array}$ & L-hydroxyproline = D-allohydroxyproline & 0.99 \\
\hline $\begin{array}{c}\text { Ribulosephosphate } \\
\text { 3-epimerase (EC 5.1.3.1.) }\end{array}$ & D-ribulose 5-phosphate = D-xylulose 5-phosphate & $1.5-3.0$ \\
\hline $\begin{array}{l}\text { UDP-glucose epimerase } \\
\text { (EC 5.1.3.2.) }\end{array}$ & UDP-glucose = UDP-galactose & 0.284 \\
\hline $\begin{array}{c}\text { Ribulosephosphate } \\
\text { 4-epimerase (EC 5.1.3.4.) }\end{array}$ & L-ribulose 5-phosphate = D-xylulose 5-phosphate & 1.86 \\
\hline $\begin{array}{c}\text { Methylmalonyl-CoA } \\
\text { racemase (EC 5.1.99.1.) }\end{array}$ & D-methylmalonyl-CoA = L-methylmalonyl-CoA & 1.0 \\
\hline $\begin{array}{c}\text { Triose phosphate isomerase } \\
\text { (EC 5.3.1.1.) }\end{array}$ & D-glyceraldehyde 3-phosphate = dihydroxyacetone phosphate & 22 \\
\hline $\begin{array}{l}\text { Arabinose isomerase } \\
\text { (EC 5.3.1.3.) }\end{array}$ & D-arabinose $=$ D-ribulose & 0.179 \\
\hline $\begin{array}{l}\text { L-arabinose isomerase } \\
\text { (EC 5.3.1.4.) }\end{array}$ & L-arabinose $=$ L-ribulose & $\sim 0.11$ \\
\hline
\end{tabular}




\section{Continued}

\begin{tabular}{|c|c|c|}
\hline $\begin{array}{l}\text { Xylose isomerase } \\
\text { (EC 5.3.1.5.) }\end{array}$ & D-xylose $=$ D-xylulose & 0.16 \\
\hline $\begin{array}{l}\text { Ribosephosphate isomerase } \\
\text { (EC 5.3.1.6.) }\end{array}$ & D-ribose 5-phosphate = D-ribulose 5-phosphate & 0.30 \\
\hline $\begin{array}{l}\text { Mannose isomerase } \\
\text { (EC 5.3.1.7.) }\end{array}$ & D-mannose $=$ D-fructose & 2.45 \\
\hline $\begin{array}{c}\text { Mannosephosphate isomerase } \\
\text { (EC 5.3.1.8.) }\end{array}$ & D-mannose 6-phosphate = D-fructose 6-phosphate & 1.78 \\
\hline $\begin{array}{c}\text { Glucosephosphate isomerase } \\
\text { (EC 5.3.1.9.) }\end{array}$ & D-glucose 6-phosphate = D-fructose-6-phosphate & 0.298 \\
\hline Glucuronate isomerase & D-glucuronate $=\mathrm{D}$-fructuronate & 0.82 \\
\hline $\begin{array}{l}\text { Arabinosephosphate isomerase } \\
\text { (EC 5.3.1.13.) }\end{array}$ & D-arabinose-5-phosphate $=$ D-ribulose 5-phosphate & 0.295 \\
\hline $\begin{array}{l}\text { L-rhamnose isomerase } \\
\text { (EC 5.3.1.14.) }\end{array}$ & L-rhamnose = L-rhamnulose & 1.5 \\
\hline $\begin{array}{c}\text { Phosphoglycerate } \\
\text { phosphomutase } \\
\text { (EC 5.4.2.1.) }\end{array}$ & 2-phospho-D-glycerate = 3-phosphoglycerate & 5.0 \\
\hline $\begin{array}{l}\text { L-methylmalonyl-CoA mutase } \\
\text { (EC 5.4.99.2.) }\end{array}$ & L-methylmalonyl-CoA = succinyl-CoA & $\sim 20$ \\
\hline $\begin{array}{l}\text { Muconate cycloisomerase } \\
\text { (EC 5.5.1.1.) }\end{array}$ & (+)-4-carboxymethyl-4-hydroxyisocrotonolactone = cis-cis-muconate & $4.03 \times 10^{-2}$ \\
\hline $\begin{array}{l}\text { Valyl-sRNA synthetase } \\
\text { (EC 6.1.1.9.) }\end{array}$ & ATP + L-valine + sRNA $=$ AMP + PPi + L-valyl-sRNA & 0.32 \\
\hline $\begin{array}{l}\text { Acetyl-CoA synthetase } \\
\text { (EC 6.2.1.1.) }\end{array}$ & $\mathrm{ATP}+$ acetate $+\mathrm{CoA}=\mathrm{AMP}+\mathrm{PPi}+$ acetyl-CoA & 0.86 \\
\hline $\begin{array}{l}\text { Acyl-CoA synthetase } \\
\text { (EC 6.2.1.2.) }\end{array}$ & $\mathrm{ATP}+$ an acid $+\mathrm{CoA}=\mathrm{AMP}+\mathrm{PPi}+$ an acyl-CoA & $\sim 1.5$ \\
\hline $\begin{array}{l}\text { Succinyl-CoA synthetase } \\
\text { (EC 6.2.1.5.) }\end{array}$ & $\mathrm{ATP}+$ Succinate $+\mathrm{CoA}=\mathrm{ADP}+\mathrm{Pi}+$ Succinyl-CoA & 0.27 \\
\hline $\begin{array}{l}\text { Glutamine synthetase } \\
\text { (EC 6.3.1.2.) }\end{array}$ & ATP + L-glutamate $+\mathrm{NH}_{3}=\mathrm{ADP}+\mathrm{Pi}+\mathrm{L}$-glutamine & $1.2 \times 10^{-3}$ \\
\hline $\begin{array}{c}\text { Adenylosuccinatesynthetase } \\
\text { (EC 6.3.4.4.) }\end{array}$ & $\mathrm{GTP}+\mathrm{IMP}+\mathrm{L}$-aspartate $=\mathrm{GDP}+\mathrm{P}_{\mathrm{i}}+$ adenylosuccinate & $2.9-10$ \\
\hline $\begin{array}{c}\text { Propionyl-CoA carboxylase } \\
\text { (EC 6.4.1.3.) }\end{array}$ & $\mathrm{ATP}+$ propionyl-CoA $+\mathrm{CO}_{2}+\mathrm{H}_{2} \mathrm{O}=\mathrm{ADP}+\mathrm{Pi}+$ methylmalonyl-CoA & 5.7 \\
\hline
\end{tabular}

reactions are usually considered non-controlling reactions in a pathway, but they can be interesting for antagonic metabolic pathways (i.e. glycolysis and gluconeogenesis), as depending on the intermediate concentrations, they can be redirected to the products or the substrates.

\section{References}

[1] Alberty, R.A., Cornish-Bowden, A., Goldberg, R.N., Hammes, G.G., Tipton, K. and Westerhoff, H.V. (2011) Reccommendations for Terminology and Databases for Biochemical Thermodynamics. Biophysical Chemistry, 155, 89103. http://dx.doi.org/10.1016/j.bpc.2011.03.007

[2] Hofmeyr, J.-H.S. and Cornish-Bowden, A. (1997) The Reversible Hill Equation: How to Incorporate Cooperative Enzymes into Metabolic Models. CABIOS, 13, 377-385.

[3] King, E.L. and Altman, C. (1956) A Schematic Method of Deriving the Rate Laws for Enzyme-Catalyzed Reactions. The Journal of Physical Chemistry, 60, 1373-1378. http://biokin.com/king-altman/index.html http://dx.doi.org/10.1021/j150544a010

[4] Barman, T.E. (1969) Enzyme Handbook. Springer-Verlag, Berlin. http://dx.doi.org/10.1007/978-3-642-86602-9 